\title{
Ultrasonido endoscópico, aplicaciones actuales en tumores sólidos gastrointestinales
}

\section{Endoscopic ultrasound: Current applications to approach gastrointestinal solid tumors}

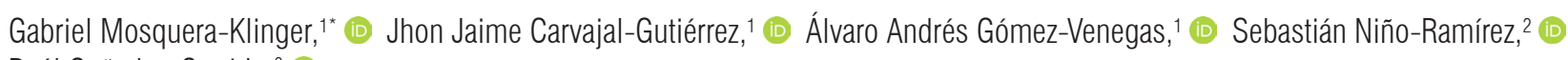
Raúl Cañadas-Garrido. ${ }^{3}$

\author{
GacCeso abierto \\ Citación: \\ Mosquera-Klinger G, Carvajal-Gutiérrez JJ, Gómez- \\ Venegas AA, Niño-Ramírez S, Cañadas-Garrido R. \\ Ultrasonido endoscópico, aplicaciones actuales en \\ tumores sólidos gastrointestinales. Rev Colomb \\ Gastroenterol. 2020;35(4):506-518. https://doi. \\ org/10.22516/25007440.521 \\ 1 Unidad de Gastroenterología y endoscopia digestiva \\ Hospital Pablo Tobón Uribe, Medellín, Colombia \\ 2 Residente de gastroenterología y endoscopia \\ digestiva, Universidad CES, Medellin, Colombia. \\ ${ }^{3}$ Gastroenterología y endoscopia digestiva, Hospital \\ Universitario San Ignacio, Bogotá. Director de \\ gastroenterología y endoscopia, Clínica de Marly, \\ Bogotá, Colombia. \\ Correspondencia: Gabriel Alonso Mosquera-Klinger \\ gami8203@yahoo.com \\ Fecha recibido: $\quad 16 / 03 / 20$ \\ Fecha aceptado: 18/05/20
}

\begin{abstract}
Resumen
La endosonografía es un método diagnóstico que viene revolucionando el abordaje de los pacientes con tumores del tracto gastrointestinal y de otros órganos y estructuras extradigestivas. En la actualidad, se viene posicionando como un método de elección en la evaluación de lesiones subepiteliales gastrointestinales y en la estadificación de muchos tumores gastrointestinales como de esófago, estómago, recto y páncreas. Por lo anterior pretendemos hacer una revisión de tema mostrando la evidencia de la endosonografía en indicaciones clásicas y aplicaciones actuales en tumores gastrointestinales.
\end{abstract}

\section{Palabras clave}

Endosonografía, estadificación de neoplasias, neoplasias gastrointestinales, neoplasias pancreáticas.

\section{Abstract}

Endosonography is a diagnostic method that has revolutionized the way to approach patients with tumors in the gastrointestinal tract and other extra-digestive organs and structures. Currently, it is a method of choice to assess subepithelial lesions of the gastrointestinal tract and to classify gastrointestinal tumors in the esophagus, stomach, rectum, and pancreas. Therefore, this literature review presents evidence on the classical indications of endosonography, as well as current applications to approach gastrointestinal tumors.

\section{Keywords}

Endosonography, Neoplasm staging, Gastrointestinal neoplasms, Pancreatic neoplasms.

\section{INTRODUCCIÓN}

El ultrasonido endoscópico (USE) es un método híbrido, en el que se utiliza un equipo de endoscopia como vehículo con un transductor de ecografía de alta resolución en su parte distal. De esta forma se obtienen imágenes endoscópicas y ecográficas con las cuales se pueden explorar lesiones dependientes de la pared gastrointestinal o extradi- gestivas aprovechando las relaciones anatómicas del tracto gastrointestinal con los órganos intraabdominales. Esta técnica nació en Japón en 1980, en 3 instituciones donde crearon el primer prototipo y fue desarrollado por la compañía Olympus Co Ltd. Este método se diseñó con el fin de crear un instrumento diagnóstico para el estudio del carcinoma pancreático pequeño (1). En Colombia se introdujo esta técnica en 1994 por el Dr. Luis Carlos Sabbagh, quien 
años más tarde, en respuesta a la necesidad de entrenamiento en países de Centro y Suramérica, fundó el Centro de formación en ultrasonido endoscópico avalado por la Organización Mundial de Gastroenterología. La utilización y aplicaciones del USE se han expandido a muchos sitios en Colombia y en la actualidad hay instituciones grandes en el país donde este método es utilizado por grupos multidisciplinarios con un enfoque diagnóstico y terapéutico.

El conocimiento y el avance en esta técnica ha crecido de forma vertiginosa. En la actualidad se dispone de equipos con mejor definición, transductores de excelente resolución utilizados con fines diagnósticos (equipo con transductor radial [Figura $\mathbf{1} \mathbf{A}$ y $\mathbf{C}$ ] y el equipo con transductor lineal [Figura 1 B y D]) que suele utilizarse para la toma de citología o biopsias con aguja fina de lesiones sólidas y para procedimientos terapéuticos como el drenaje de colecciones y la ablación de tumores sólidos, entre otras aplicaciones.
La Asociación Americana de Gastroenterología considera que tiene privilegios o competencias para realizar este procedimiento un médico gastroenterólogo o cirujano gastrointestinal, que realice un entrenamiento Hands-on en un centro de enseñanza que cuente con un número adecuado de procedimientos/año, además de que el estudiante tenga una experiencia suficiente en la colangiopancreatografía retrograda endoscópica (CPRE). En relación con las competencias para poder realizar con seguridad este procedimiento, diferentes sociedades científicas como la Sociedad Americana de Gastroenterología y Endoscopia Digestiva (ASGE), la Sociedad Europea de Gastroenterología y Endoscopia (ESGE) y la Sociedad Británica de Gastroenterología (BSG) recomiendan que durante el entrenamiento el estudiante realice entre 225250 procedimientos supervisados por un experto y, de estos, entre 50 y 75 punciones $(2,3)$.
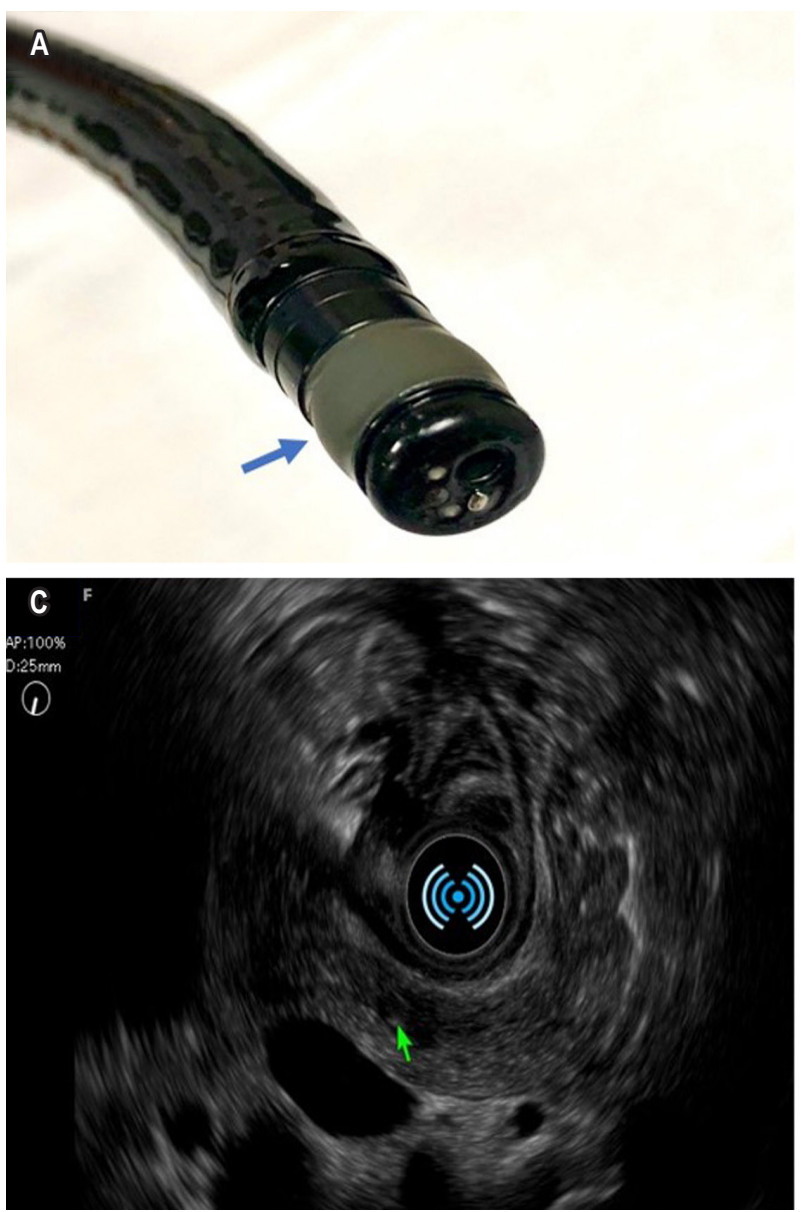

B

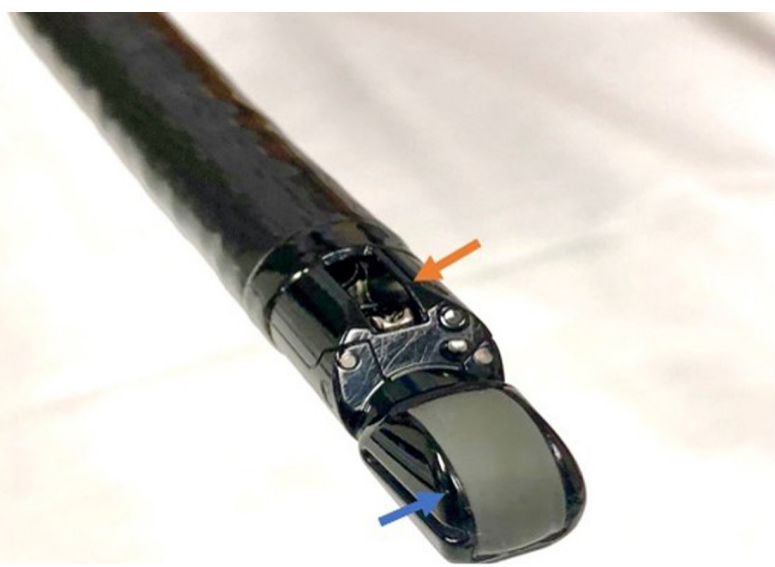

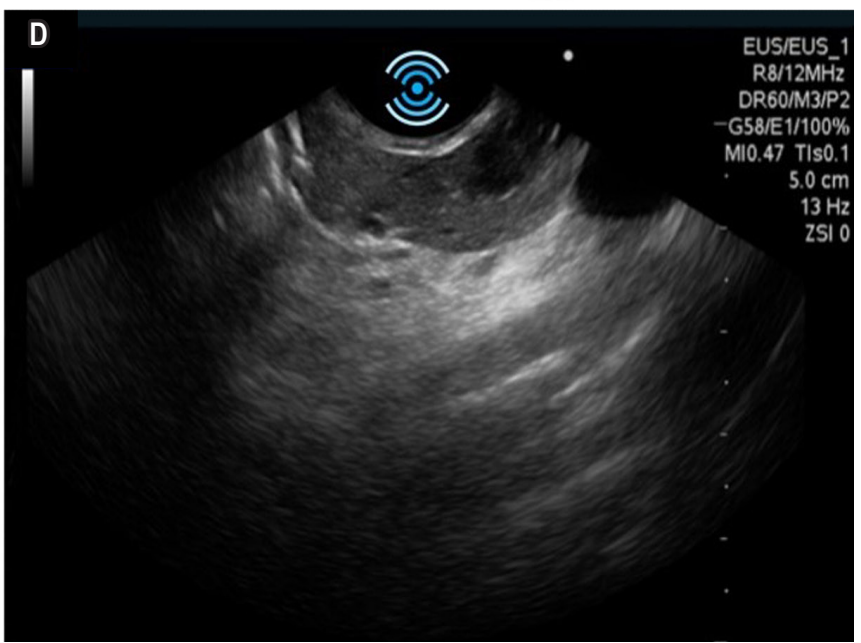

Figura 1. A. Equipo con transductor radial: la visión endoscópica es frontal, la imagen sonográfica es radial; la flecha azul señala el transductor radial. B. Transductor lineal: visión endoscópica oblicua, visión sonográfica sectorial; la flecha azul señala el transductor y la flecha naranja señala el canal de trabajo que queda en disposición perpendicular en relación con el transductor. C. Visión sonográfica radial, documentación de una lesión hipoecoica ovalada en el cuello del páncreas compatible con tumor neuroendocrino. D. Visión con transductor lineal de la misma lesión en el cuello del páncreas. 
La endosonografía es un método seguro en manos entrenadas. En los procedimientos diagnósticos se describe que las complicaciones son similares a la endoscopia digestiva superior. En los casos terapéuticos que se realizan con el transductor lineal podría aumentar el riesgo de perforación en el cricofaríngeo hasta 2 veces respecto a la endoscopia convencional debido a la mayor rigidez, el mayor diámetro del equipo y la visión endoscópica oblicua $(4,5)$. Además, los riesgos son inherentes a la diana o lesión/colección a puncionar. Los eventos descritos con más frecuencia son perforación $(0,03 \%)$, sangrado $(0,13 \%)$, pancreatitis aguda en punciones del páncreas (en el rango de $0 \%$ a $2 \%$ ) y la peritonitis biliar es extremadamente rara (escasos reportes de casos) (6).

\section{APLICACIONES ACTUALES DEL USE}

\section{Evaluación de lesiones subepiteliales (LSE)}

Las lesiones subepiteliales son tumores que se originan de capas profundas correspondientes a la capa muscular de la mucosa, capa submucosa o capa muscular propia del tracto digestivo. Son más frecuentemente observadas en el estómago, donde se podrían identificar en una de 1 de cada 300 endoscopias realizadas (6). Se identifican como elevaciones o protrusiones de la mucosa, la mayoría son menores de $2 \mathrm{~cm}$ de diámetro, suelen identificarse en la realización de exámenes endoscópicos de rutina en forma incidental. En algunos casos estas lesiones se pueden presentar en el contexto de sangrado, obstrucción o metástasis. La importancia del estudio de las LSE es que la apariencia endoscópica de dichas lesiones puede ser similar y hasta un $15 \%$ de estas pueden ser malignas o con riesgo potencial de malignización (7). La endosonografía se considera como la técnica más precisa para la evaluación de lesiones subepiteliales debido a su capacidad de definir con certeza las capas histológicas y, por tanto, el sitio más probable de origen del tumor (Tabla 1). Además, es superior a otras modalidades de imágenes como la tomografía axial computarizada (TAC) o la resonancia magnética (RMN) en la caracterización de lesiones pequeñas $(<2 \mathrm{~cm})(7)$, ya que puede distinguir con precisión entre la compresión extrínseca del tracto gastrointestinal y un crecimiento intramural.

En las lesiones de la pared gastrointestinal se logran identificar las 5 ecocapas principales: la primera y segunda (mucosa que incluye la capa muscular de la mucosa), la tercera (submucosa), la cuarta (la capa muscular propia) y la quinta (serosa o adventicia) (Figura 2 A). También permite la medición del tamaño de la lesión y la evaluación de cualquier linfadenopatía asociada para su posterior estadificación. En el grupo de lesiones que tienen un riesgo potencial de malignizarse se encuentra el tumor del estroma gastrointestinal (GIST) y la mayoría de estos son gástricos (constituye entre el $60 \%$ y $70 \%$ de todos los casos) (Figura 2 B), $20 \%-30 \%$ se ubican en el intestino delgado y un poco menos del $5 \%$, en el esófago. Respecto al diagnóstico de GIST, el objetivo de la obtención de tejido es recoger material que demuestre células fusiformes, además de la realización de inmunohistoquímica (IHQ). En la IHQ se recomienda realizar las tinciones de CD117, DOG1, S100, CD34 y PDGFRA; con estas se logra diferenciar de forma adecuada el GIST de otras lesiones subepiteliales (8). Tradicionalmente, la punción/aspiración con aguja fina USE-guiada (USE-PAAF) ha sido el método para obtener
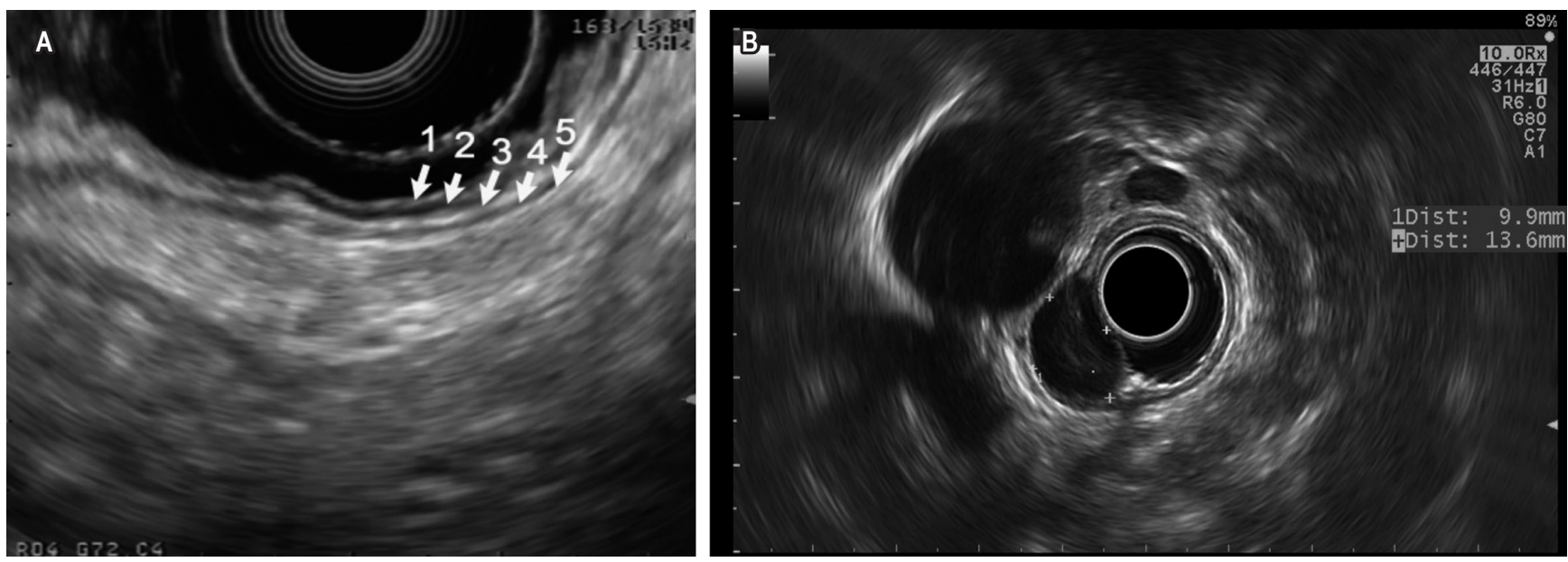

Figura 2. A. Visión endosonográfica con transductor lineal de las ecocapas de la pared gástrica normal. B. Lesión ovalada hipoecoica homogénea correspondiente a GIST de la cuarta ecocapa, evaluada con transductor radial. Imágenes cortesía del Dr. Cañadas, Clínica Marly. 
Tabla 1. Características endoscópicas y endosonográficas de las lesiones subepiteliales en el tracto digestivo

Lesión subepitelial

Lesiones benignas

- GIST

- Leiomioma

- Lipoma

- Várices

- Origen neural (schwannoma, Inespecífica neuroma, neurofibroma)

- Tumor de células granulosas

- Pólipo fibroide inflamatorio

Inespecífica, solitarias, menores de $4 \mathrm{~cm}$

Con bordes lisos, solitaria, polipoide, con ulceración mucosa, tamaño entre 2 y $5 \mathrm{~cm}$

- Quiste de duplicación

\section{Apariencia regular, con bordes lisos y} traslúcidos, compresible

\begin{tabular}{ll} 
- Linfangioma & $\begin{array}{l}\text { Masa de aspecto pseudoquístico, } \\
\text { comprensible, comúnmente en el intestino }\end{array}$ \\
$\begin{array}{ll}90 \% \text { umbilicados, > } 90 \% \text { está localizado } \\
\text { en el antro }\end{array}$ \\
$\begin{array}{ll}\text { - Hiperplasia de las glándulas } \\
\text { de Brunner }\end{array}$ & Bulbo duodenal \\
\hline
\end{tabular}

Ecocapa

Apariencia en el USE

4 (raramente 2 o 3) Hipoecoica, mayormente $<3-5 \mathrm{~cm}$, redonda, homogénea

Hipoecoica, homogénea, bien circunscrita

2,304

Intensamente hiperecoica, homogénea, con bordes lisos

Anecoica, serpiginosa, Doppler positivo

Hipoecoica

203

203

Hipoecoica, con ecotextura heterogénea

304

Hipo- a hiperecoica, homogéneas

Cualquiera o extramural

Anecoica, con refuerzo ecogénico posterior, entre 3 y 5 ecocapas, redonda u ovalada, Doppler negativo

Tercera Anecoica con tabique interno

2,304

203

Hipoecoica o con ecogenicidad mixta, bordes no claros, con una área anecoica o tubular

Hiperecoica, área anecoica, con bordes lisos

Lesiones con potencial riesgo de malignización

$\begin{array}{llll}\text { - GIST } & \text { Lesión ulcerada } & 4 \text { (raramente 2 0 3) } & \begin{array}{l}\text { Hipoecoica, }>3 \mathrm{~cm} \text {, bordes irregulares } \\ \text { extraluminales, espacios quísticos, } \\ \text { heterogénea, con focos ecogénicos }\end{array} \\ \text { - Neoplasia neuroendocrina } & \begin{array}{l}\text { Inespecífica, podría ser de apariencia } \\ \text { amarilla; los carcinoides gástricos podrían } \\ \text { ser múltiples }\end{array} & 203 & \begin{array}{l}\text { Levemente hipoecoica o isoecoica, } \\ \text { homogénea, ovalada, con bordes lisos }\end{array} \\ \text { - Linfoma } & \text { Inespecífica } & 2,304 & \text { Hipoecoica heterogénea } \\ \text { - Metástasis } & \text { Inespecífica } & \text { Cualquier ecocapa } & \text { Hipoecoica, masa heterogénea } \\ \text { - Tumor glómico } & \text { Inespecífica, más frecuente en el antro } & 304 & \begin{array}{l}\text { Hipo- o hiperecoica. Más de la mitad de casos } \\ \text { con puntos hiperecoicos, Doppler positivo }\end{array}\end{array}$

GIST: tumor del estroma gastrointestinal. USE: ultrasonido endoscópico. Adaptado de: Standards of Practice Committee et al. The role of endoscopy in subepithelial lesions of the GI tract. Gastrointest Endosc. 2017;85(6):1117-1132.

tejido. El rendimiento diagnóstico es muy variable (entre $46 \%$ y $93 \%)$ y, en general, es limitado porque el tamaño de muestra podría ser insuficiente para realizar IHQ. En un estudio multicéntrico reciente se compararon la biopsia con aguja fina (USE-BAAF) frente al USE-PAAF en el ren- dimiento en la obtención de muestras para citopatología, en la capacidad de obtener un diagnóstico basado en IHQ y el rendimiento diagnóstico definitivo. Los resultados en muestras de citopatología fueron de $92 \%$ frente a $46 \%(p$ $=0,001), 89 \%$ frente a $41 \%$ en IHQ $(p=0,001)$ y $89 \%$ 
frente a $37 \%$ en diagnóstico definitivo $(p=0,001)$ entre los grupos BAAF frente a PAAF, respectivamente. Los autores concluyen que el USE-BAAF es superior al USE-PAAF en el rendimiento diagnóstico histológico del GIST (9).

En un estudio publicado recientemente, se observó una precisión general del USE del 64,2\%, en comparación con la TAC, del 50,9 \%. En particular, respecto a la precisión del USE frente a la TAC para GIST, leiomiomas y páncreas ectópico, los resultados fueron de $83,9 \%$ frente a $74,2 \%$, $37,5 \%$ frente a $0,0 \%$, y $57,1 \%$ frente a $14,3 \%$, respectivamente. La mayoría de los diagnósticos incorrectos en USE se refería a lesiones hipoecoicas originadas en la cuarta ecocapa, y las lesiones mal diagnosticadas más comunes son el GIST que se confunde con leiomioma y viceversa (10).

Otra indicación actual del USE es en la caracterización de lesiones subepiteliales, en las que se evalúa su profundidad para definir resecciones endoscópicas. En la Figura 3 se observa un tumor neuroendocrino de la segunda ecocapa donde se realiza mucosectomía con fines curativos.

\section{Diagnóstico y estadificación de tumores de esófago}

El cáncer de esófago es la sexta causa de cáncer en el mundo, se diagnostica generalmente en pacientes con síntomas de reflujo de larga data o con disfagia, a quienes se les realiza una endoscopia con toma de biopsias. En ocasiones, el diagnóstico se realiza mediante estudios imagenológicos como la TAC de abdomen en el escenario de dolor abdominal o de pérdida anormal de peso o con menor frecuencia como hallazgo incidental. Por esta razón, la mayoría de veces el diagnóstico de cáncer de esófago se realiza en estadios avanzados.

En la actualidad, para la estadificación de los tumores esofágicos y de la unión gastroesofágica se utiliza la clasificación de la Unión Internacional para el Control del Cáncer (UICC) y la clasificación TNM (tumor, ganglios, metástasis) que se encuentra actualmente en su 8.a edición. De los cambios de interés entre la 7. ${ }^{\mathrm{a}}$ y $8 .^{\mathrm{a}}$ edición de TNM es la reclasificación de los estadios. El estadio IIIB y IIIC (T3-4a N1-3) de la séptima edición se reclasificaron como estadio IVA, y la enfermedad M1 se clasifica como estadio IVB. Estos pacientes tienen un mal pronóstico, tan pobre como los individuos con enfermedad metastásica. Otro cambio en la 8.a edición de TNM es la separación de los tumores $\mathrm{T} 1$ en tumores T1a y T1b. Esta diferenciación es importante porque el riesgo de metástasis ganglionares aumenta del $3 \%$ al $6 \%$ para los tumores de la mucosa T1a, al 21,24\% para los tumores submucosos $\mathrm{T} 1 \mathrm{~b}$ (11). Esta diferenciación de los tumores $\mathrm{T} 1$ es importante para guiar las decisiones sobre el tratamiento endoscópico frente al quirúrgico (12) y, en este escenario, el papel del USE es fundamental. La estadificación inicial se podría hacer con TAC de tórax y abdomen contrastado para definir si la enfermedad es irresecable o identificar metástasis a distancia. Si se considera que el paciente tiene una enfermedad potencialmente curable, entonces se recomienda la tomografía por emisión de positrones (PET scan), seguida de la endosonografía. La laparoscopia diagnóstica debe realizarse solo en pacientes con un tumor esofágico que se extienda al estómago.

El orden óptimo de realización de las investigaciones es discutible, aunque en general se está de acuerdo con que el PET scan debe realizarse antes que el USE. La enfermedad incurable a distancia detectada en la PET scan evitaría que un paciente se sometiera a el USE, lo que disminuye riesgos $y$ costos relacionados con el procedimiento. Se ha descrito que el USE influye en las decisiones de tratamiento en el $29 \%$ de los pacientes, principalmente en el diagnóstico de metástasis ganglionares y en la definición del volumen tumoral bruto durante la planificación de la radioterapia (12). Se ha demostrado que las mediciones del tumor con PET scan y de la longitud de la enfermedad difieren significativamente, y que tiende a producir menores dimensiones (13). En conclusión, el PET scan es el método de elección para la metástasis, aumentando en un $38 \%$ la eficiencia diagnóstica con respecto a la TAC (14).
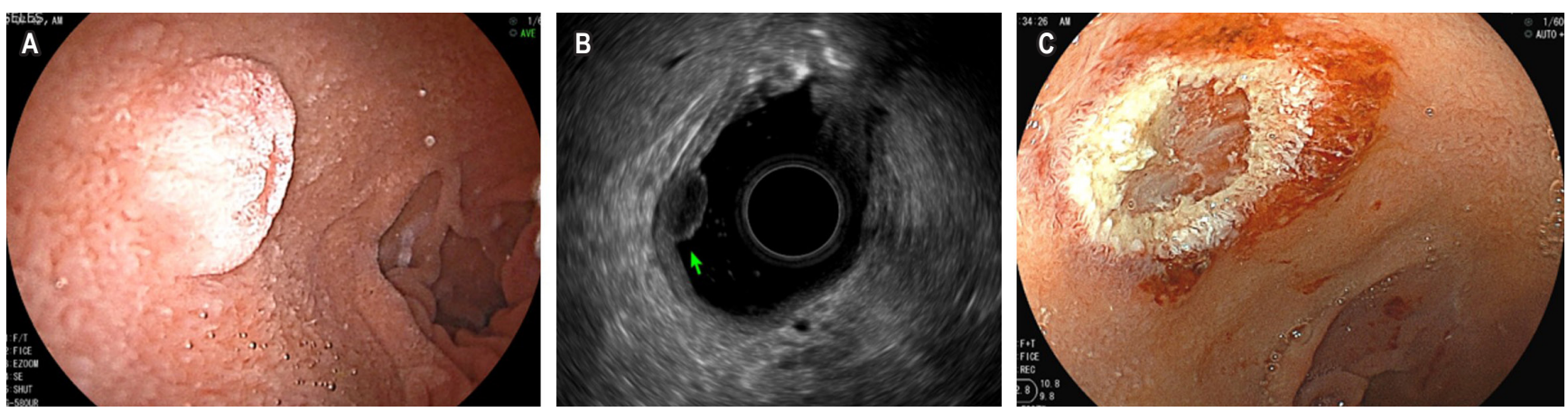

Figura 3. A. Visión endoscópica de un tumor neuroendocrino en el bulbo duodenal. B. Visión sonográfica. C. Resección mediante mucosectomía. Imágenes cortesía del Dr. Carvajal, Hospital Pablo Tobón Uribe (HPTU). 
En general, se considera que el USE actualmente es el estándar de oro para la evaluación de la etapa locorregional, ya que es el método de elección para la T y la N (Figura 4) (12). Además, tiene la ventaja sobre otros métodos por la posibilidad de realizar punciones o biopsias con aguja fina (aumentado así la especificidad). Se describen 3 limitaciones: que es un procedimiento dependiente del operador, que en la mayoría de casos se requiere sedación y los riesgos inherentes a la técnica y la imposibilidad de pasar la lesión en caso de tumores estenosantes. En este último aspecto, se describe que la falta en el paso de la estenosis tumoral se presenta aproximadamente en el $30 \%$, estas cifras se reducen hasta el $3 \%$ usando minisondas (15).

\section{Diagnóstico y estadificación de tumores gástricos}

El cáncer gástrico se considera el cuarto cáncer más prevalente en el mundo y el tercero en Colombia. Este es considerado el segundo cáncer con mayor mortalidad en el mundo. La detección temprana impacta de forma significativa en el pronóstico, el problema más importante es que la mayoría de pacientes suelen consultar en estadios avanzados de la enfermedad. Para dimensionar el impacto que tiene el momento del diagnóstico, la supervivencia a 5 años en países europeos es entre $10 \%$ y $30 \%$, mientras que en Japón (donde se hacen campañas masivas de tamización) la supervivencia a 5 años es tan alta como un $90 \%$ (16).

El USE es el mejor método disponible para evaluar los tumores y ganglios. Este tiene mayor rendimiento que la TAC y que la RMN para los ganglios, especialmente porque se pueden obtener muestras mediante punción o biopsia por aspiración con aguja fina, pero no es muy útil para evaluar la metástasis, por lo que habitualmente se solicita esta herramienta como complemento del PET scan, RMN o TAC (17).

En un metaanálisis de 4397 pacientes se pudo establecer la utilidad de la endosonografía para discriminar los carcinomas gástricos superficiales ( $\mathrm{T} 1$ a $\mathrm{T} 2$; con un radio bajo la curva de 0,86 ) de los avanzados ( $\mathrm{T} 3$ a $\mathrm{T} 4$; con un radio bajo la curva de 0,9). En términos generales, la capacidad de la endosonografía para establecer si se trata de un adenocarcinoma limitado a la mucosa o de un cáncer gástrico invasor se da con una sensibilidad y especificidad de 0,85 (intervalo de confianza [IC] $95 \%: 0,78$ a 0,91 ) y 0,90 (IC $95 \%: 0,85$ a 0,93$)$, respectivamente. En este estudio se pudo establecer que, a pesar del buen rendimiento diagnóstico, la razón de probabilidad positiva y negativa no fue suficiente para recomendarlo como examen único, por lo cual habitualmente se solicita esta herramienta como complemento del PET scan, RMN o TAC (18).

En un estudio europeo en el que se comparó la endosonografía frente a la TAC multidetector (TACMD) en cuanto al rendimiento diagnóstico en la estadificación locorregional del adenocarcinoma gástrico, se evaluaron 77 pacientes quirúrgicos con adenocarcinoma gástrico, de los cuales 42 tuvieron una estadificación preoperatoria completa y, finalmente, fueron incluidos en el estudio. Con respecto a la precisión general de la estadificación $\mathrm{T}$, la endosonografía fue superior al TACMD (62\% frente a $50 \%)$. En un subanálisis de estadios tempranos (T1-T2) y avanzados (T3-T4), la precisión y sensibilidad fueron mayores para el USE ( $83,3 \%$ frente a
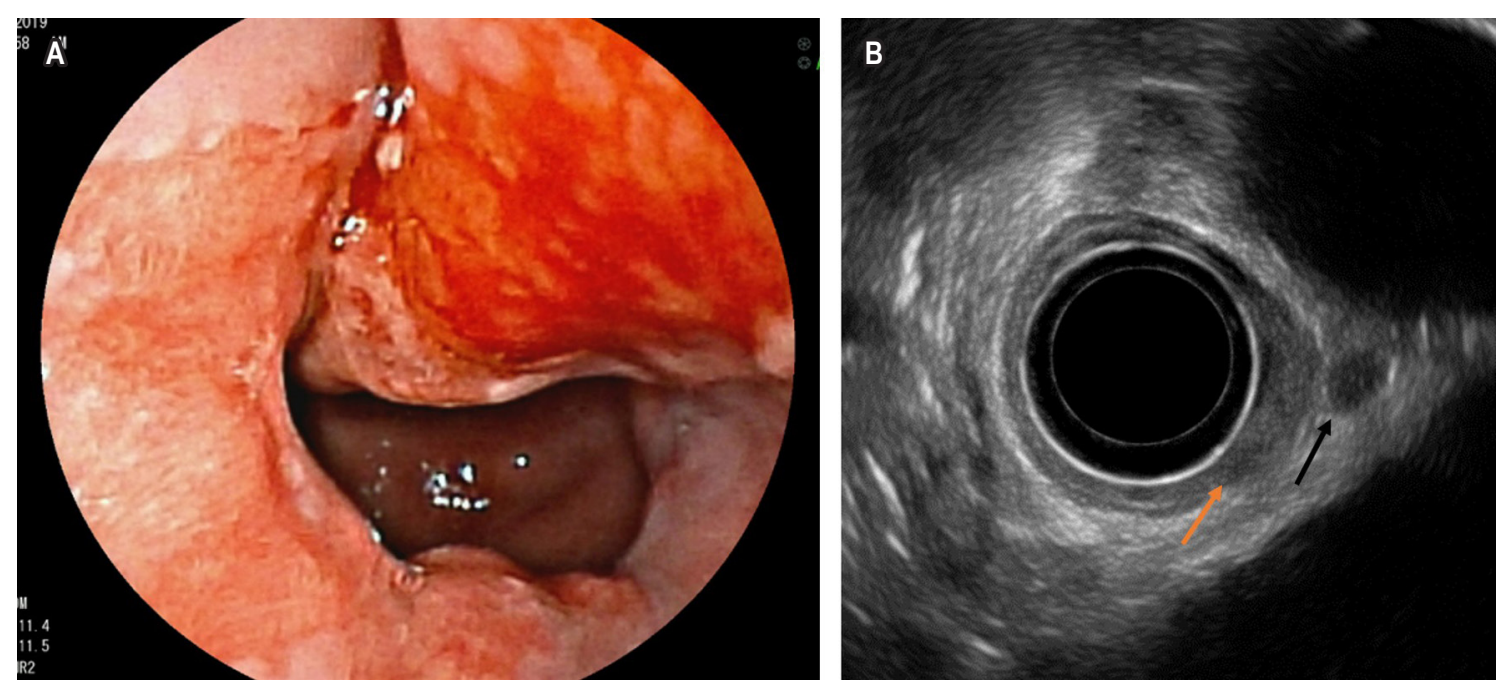

Figura 4. A. Visión endoscópica de una lesión neoplásica proveniente de un esófago de Barrett. B. Visión sonográfica de engrosamiento mucoso con compromiso transmural (flecha naranja) y adenopatía perilesional (flecha negra). 
$64,29 \%$ y $84,4 \%$ frente a $59,5 \%$, respectivamente), aunque esto no alcanzó a ser estadísticamente significativo. La precisión y sensibilidad general del USE para la estadificación de la $\mathrm{N}$ fue menor que la de la TACMD, aunque ninguna de las comparaciones alcanzó a ser estadísticamente significativa ( $57 \%$ frente a $64 \%$ y $29 \%$ frente a $55 \%$ ). Por lo anterior se concluyó que el rendimiento diagnóstico del USE es similar al de la nueva TACMD con respecto a la estadificación preoperatoria de $\mathrm{T}$ y $\mathrm{N}$ del adenocarcinoma gástrico; sin embargo, ambas técnicas deben considerarse complementarias hasta que estudios más grandes y aleatorios puedan confirmar estos resultados (19).

\section{Diagnóstico en tumores colorrectales: pólipos rectales de alto riesgo}

En lesiones rectales polipoides no pediculadas mayores a $2 \mathrm{~cm}$ de diámetro es importante establecer la probabilidad de invasión a capas profundas para definir la mejor técnica de resección (endoscópica mediante mucosectomía frente a disección endoscópica de la submucosa o técnicas quirúrgicas mínimamente invasivas). Algunos expertos sugieren realizar imágenes complementarias como el USE para definir la técnica de resección ideal en lesiones de alto riesgo como en displasia de alto grado, 0-IIa+c de París, extensión lateral no granular, patrón de Kudo $\mathrm{V}$ o mayores a $3 \mathrm{~cm}$ (20). Las lesiones con invasión profunda de la submucosa (sm) tienen un riesgo elevado de metástasis ganglionar (sm1 del $2 \%$, sm 2 del $8 \%$ y sm 3 del $23 \%$ ); por tanto, su manejo debe ser quirúrgico. En este contexto, la RNM tiene un rendimiento variable para definir el grado de inva- sión de las lesiones polipoides en el recto, particularmente en lesiones polipoides T1 ( $\mathrm{pT} 1$ ), en las que la precisión de la profundidad de invasión es variable, desde un $25 \%$ hasta un $98 \%$ (21). En un metaanálisis de 42 estudios con 5309 pacientes, se encontró una sensibilidad y especificidad del USE para la estadificación de lesiones rectales T1 del $88 \%$ y $98 \%$ respectivamente; y del $80 \%$ y $96 \%$, respectivamente, en lesiones T2. Es por esto que el USE se sugiere como la mejor estrategia diagnóstica para abordar lesiones tempranas ( $\mathrm{pT} 1)$, mientras que la RNM se recomienda para valorar lesiones pT2 o más avanzadas (22).

La Sociedad Europea de Radiología Abdominal y Gastrointestinal (ESGAR), en su guía para el estudio de neoplasias de recto, sugiere que el USE es el método de elección para la diferenciación y estadificación de lesiones T1 rectales (Figura 5) (23). Se ha descrito que, en las lesiones polipoides del recto, el USE reduce el riesgo de carcinoma no diagnosticado del $21 \%$ al $3 \%$, al compararse con la endoscopia convencional (24). En este contexto, se ha comenzado a valorar el uso de la elastografía por USE para medir las propiedades elásticas como la dureza del tejidoy, de este modo, poder diferenciar entre pólipos benignos y malignos rectales. Un estudio demostró que la elastografía podría diferenciar adenomas benignos de adenocarcinoma invasivo con una sensibilidad del $96 \%$, especificidad del $86 \%$ y una precisión diagnóstica del $94 \%$ al corroborarse con la patología (25).

\section{Cáncer de recto}

La adecuada estadificación del cáncer de recto es crucial para establecer el pronóstico y el manejo más apropiado
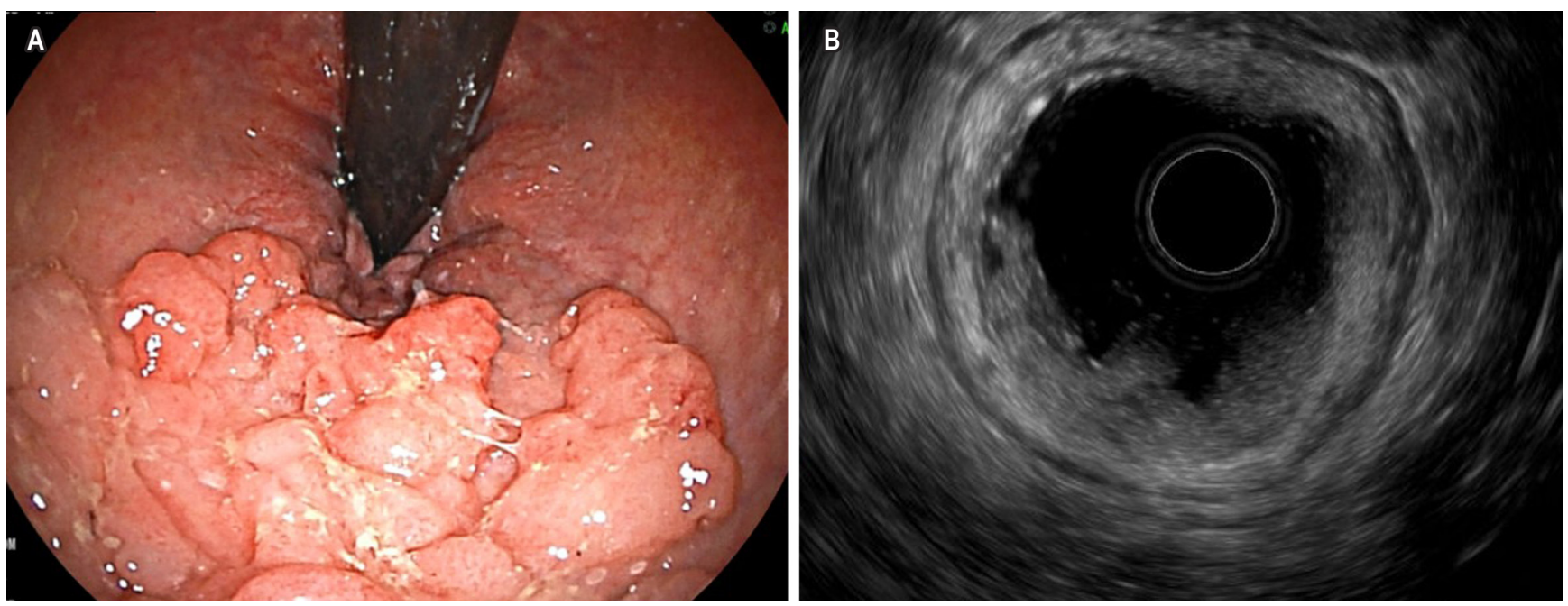

Figura 5. A. Visión endoscópica en retroflexión de una lesión con extensión lateral en el recto distal. B. Lesión mucosa, con extensión hasta la submucosa (uT1 sm). Imagen cortesía del Dr. Carvajal, HPTU. 
para el paciente. Cuando se utiliza como método de estadificación en cáncer de recto para definir la $\mathrm{T}$ (tumor), se propone la clasificación uT (Tabla 2) (26). El USE podría tener la ventaja en estadificar la $\mathrm{T}$ en lesiones tempranas (T1), pero su rendimiento es inferior a la RNM en cuanto a la precisión en la estadificación de lesiones $\mathrm{T} 2$ en adelante (22). Un metaanálisis publicado en 2004 demostró un rendimiento diagnóstico similar en la estadificación de la $\mathrm{N}$ (ganglios) en tumores de recto para el USE y la RNM con una sensibilidad y especificidad del $67 \%$ y $78 \%$ y del $66 \%$ y $76 \%$, respectivamente $(27)$.

Tabla 2. Estadificación por USE del cáncer rectal (clasificación uT)

\begin{tabular}{|c|l|}
\hline uT1 & $\begin{array}{l}\text { Tumor con invasión limitada a la mucosa y submucosa } \\
\text { T1m si infiltra solamente mucosa, con una capa muscular } \\
\text { de la mucosa preservada } \\
\text { T1sm cuando hay invasión de la submucosa }\end{array}$ \\
\hline uT2 & $\begin{array}{l}\text { Tumor con infiltración de la capa muscular propia, } \\
\text { extendiéndose a través de las primeras } 4 \text { ecocapas de la } \\
\text { pared rectal. La capa externa, que corresponde a la capa } \\
\text { muscular propia, es lisa, lo que sugiere que el tumor está } \\
\text { limitado a la pared rectal. }\end{array}$ \\
\hline uT3 & $\begin{array}{l}\text { Tumor con invasión de la grasa perirrectal, con irregularidad } \\
\text { de la cuarta ecocapa, que sugiere diseminación tumoral por } \\
\text { fuera de la pared rectal. }\end{array}$ \\
\hline uT4 & $\begin{array}{l}\text { Tumor con infiltración a los órganos y estructuras } \\
\text { adyacentes. }\end{array}$
\end{tabular}

Modificado de: Marone P et al. Role of endoscopic ultrasonography in the loco-regional staging of patients with rectal cancer. World J Gastrointest Endosc. 2015;7(7):688-701.

Las guías del National Comprehensive Cancer Network (NCCN) en su versión actualizada del 2020 sugiere que, en las lesiones polipoides con focos de carcinoma invasivo llevadas a resección endoscópica y cuya muestra histopatológica esté fragmentada, con bordes no definidos o con histología no favorable, se debe realizar RNM como primer estudio de estadificación previo a llevar resección quirúrgica ya sea transanal o transabdominal. Esta guía sugiere la realización del USE si la RNM está contraindicada o para lesiones superficiales (28). Para la estadificación inicial de tumores de recto, de igual manera, sugieren como primer estudio la RNM pélvica y se reserva el USE en pacientes con RNM contraindicada. En cuanto al seguimiento de pacientes llevados a resección quirúrgica transanal, sugieren evaluar la anastomosis con USE o RNM cada 3 a 6 meses por los primeros 2 años y luego cada 6 meses por 5 años para descartar recaídas locales $(28,29)$.

\section{Diagnóstico y estadificación de tumores sólidos biliopancreáticos}

El cáncer de páncreas es altamente letal, es la cuarta causa de muerte por cáncer en Estados Unidos. El tratamiento quirúrgico mediante una duodenopancreatectomía con técnica de Whipple es una opción curativa, pero solo el $15 \%$ de los pacientes es candidato en el momento de presentación. En estadios avanzados, la supervivencia con quimioterapia es 8,5 meses (30). El USE ha sido un método que ha venido ganando terreno en el abordaje de patologías biliopancreáticas, con una precisión diagnóstica que varía entre el $78 \%$ y $98 \%$. El rendimiento diagnóstico del USE aumenta cuando se adiciona PAAF o BAAF (lesión sólida en la cabeza de páncreas en la que se realizó una PAAF, Figura 6).

La precisión diagnóstica está influenciada por factores propios de la lesión como la localización, tamaño y tipo; de aspectos técnicos como el número de pases; la técnica utilizada para el muestreo (aspiración, técnica de slow pull, técnica de Fanning); la experiencia del endoscopista; y
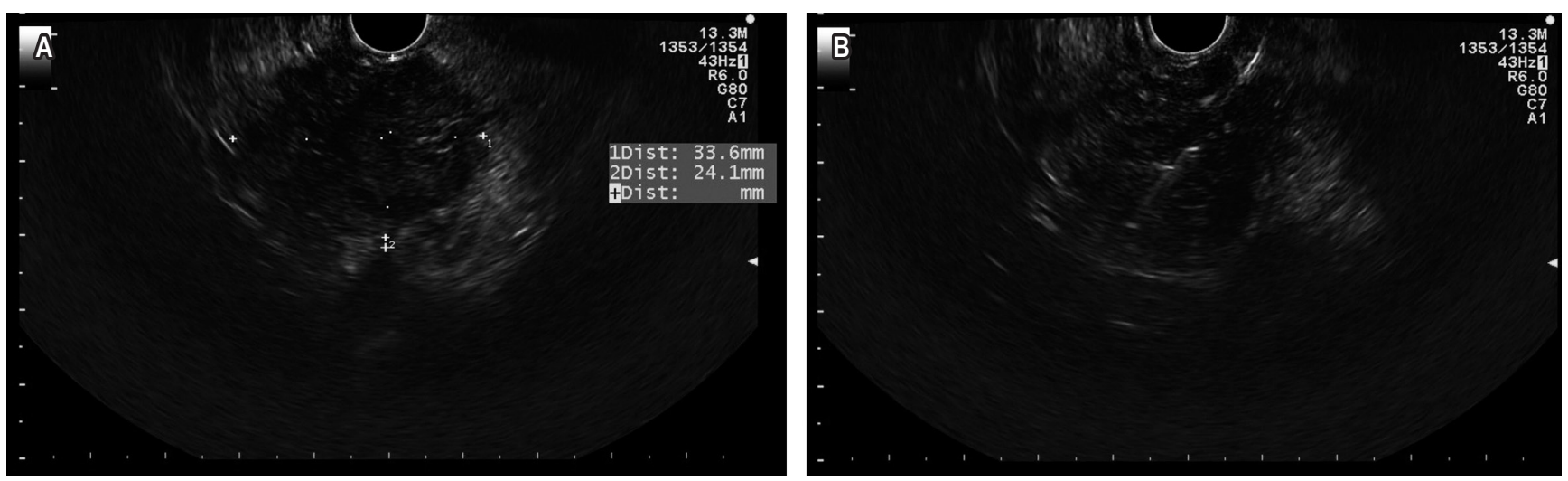

Figura 6. A. Lesión sólida en la cabeza del páncreas. B. PAAF de la lesión. Imagen cortesía del Dr. Cañadas, Clínica Marly. 
entre los factores menos estudiados está la presencia de patólogo en sala.

El USE-PAAF es el método de elección para la toma de muestras de lesiones sólidas pancreáticas (30-32). Se considera que es más sensible que la TAC de abdomen y que la RMN en lesiones menores de $10 \mathrm{~mm}$ de tamaño. Además, es un método seguro y costo-efectivo ya que provee un alto rendimiento diagnóstico (33). Consideramos que una de las claves para obtener los mejores resultados en relación con las punciones por USE es obtener un material suficiente/representativo de la lesión y transferir adecuadamente al laboratorio de patología. En algunos centros de referencia mundial se ha realizado el USE-PAAF con patólogo en sala, esto ha permitido disminuir el número de pases $y$, en general, aumentar el rendimiento de la prueba.
Recientemente, el grupo de trabajo del HPTU de Medellín mostró su experiencia preliminar de las punciones y biopsias biliopancreáticas con patólogo en sala (Figura 7). En este trabajo se resalta que dicha estrategia permitió obtener una alta eficiencia diagnóstica (cercana al $90 \%$ ), con escasos falsos negativos (34).

\section{APLICACIONES EN DESARROLLO}

\section{Ablación guiada por endosonografía de tumores neuroendocrinos (insulinoma)}

El insulinoma pancreático esporádico es un tumor benigno en las células $\beta$ de los islotes de Langerhans, generalmente solitario y menor de $20 \mathrm{~mm}$. Cuando es funcionante, los
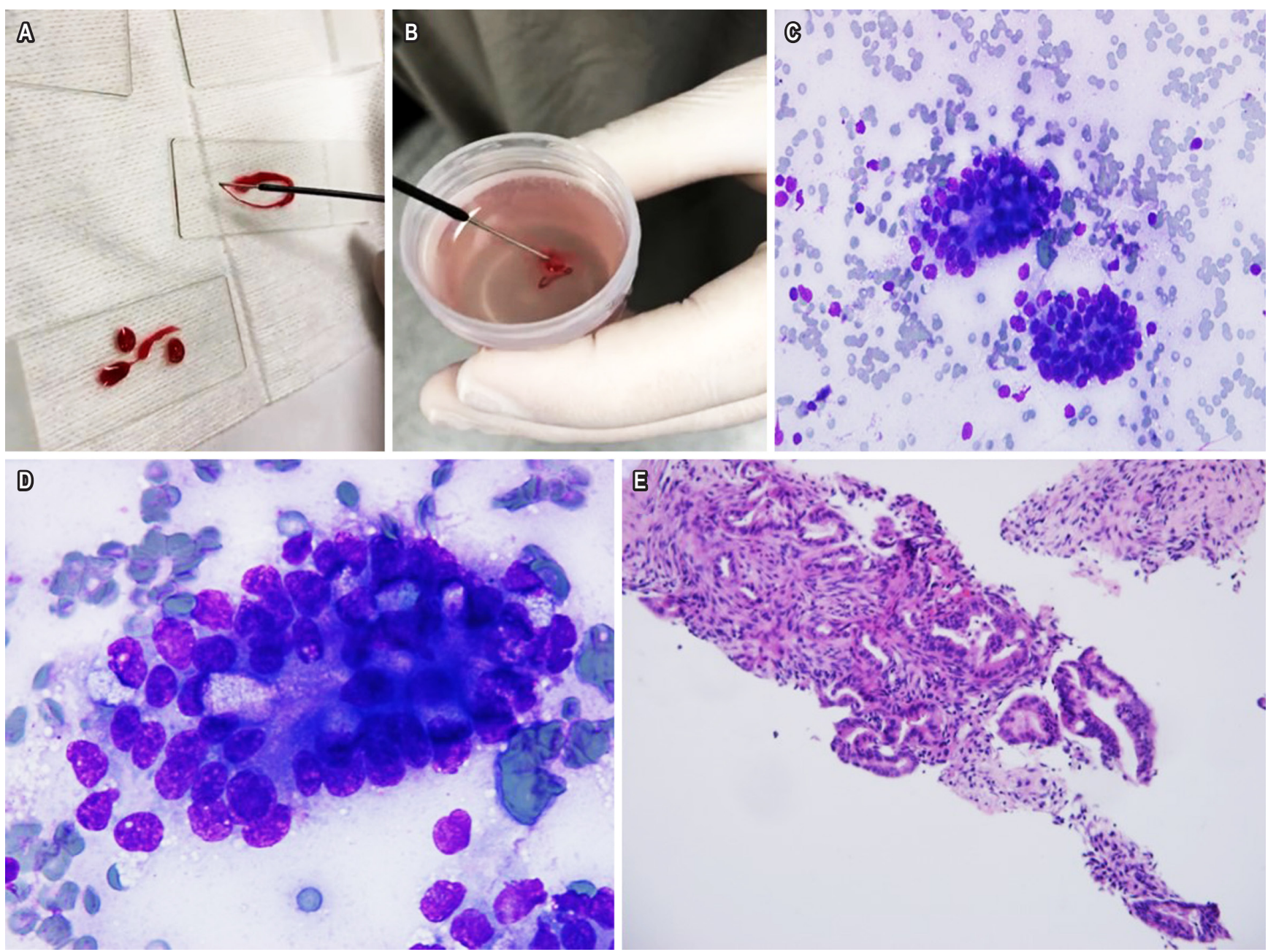

Figura 7. A. Extendido de tejido pancreático en sala de endoscopia. B. BAAF colocada en un frasco con formol. C. Citología con Diff-Quick 40x: evidencia de adenocarcinoma ductal de páncreas. D. Citología Diff-Quick 100x con adenocarcinoma ductal.E. BAAF con evidencia de adenocarcinoma ductal. Imagen cortesía del Dr. Mosquera-Klinger, HPTU. 
pacientes experimentan síntomas relacionados con la producción excesiva de insulina. Se describe la triada de Whipple (síntomas de hipoglucemia, hipoglucemia venosa, desaparición de síntomas con la corrección en los niveles de glucemia). El diagnóstico es clínico, con confirmación bioquímica y radiológica. El tratamiento suele ser quirúrgico, ya sea mediante duodenopancreatectomía con técnica de Whipple, pancreatectomía distal o total, o enucleación según el tamaño y la localización.

En 1999 se describió la ablación por radiofrecuencia USEguiada en modelos porcinos (35); posteriormente, se iniciaron a describir casos de tratamientos en lesiones sólidas pancreáticas como una medida paliativa en pacientes con alto riesgo quirúrgico (36-38). En la actualidad, se está desarrollando la ablación USE-guiada como tratamiento de insulinomas pancreáticos en pacientes con múltiples comorbilidades que no sean candidatos a cirugía o quienes rechacen la cirugía (39-43). En Colombia, realizamos los 2 primeros casos de ablación mediante etanolización en 2 mujeres que debutaron con coma en el contexto de hipoglucemia hiperinsulinémica y elevación concomitante del péptido C. En ambos casos, la RMN de abdomen fue reportada como normal y asociada con hallazgos endosonográficos compatibles con tumor neuroendocrino. Resaltamos que en ambos casos se resolvió de forma inmediata la hipoglucemia después de la ablación, sin complicaciones y con una mejoría completa de sus síntomas (en el seguimiento a 12 meses) (44).

\section{Punciones esplénicas por USE}

El estudio histológico de las lesiones focales esplénicas o de la esplenomegalia de origen incierto ha sido limitado por el riesgo que supone la realización de punciones a nivel esplénico. El evento adverso más importante descrito es el sangrado (hemoperitoneo o hematoma capsular), aunque también se pueden presentar lesiones de estructuras vecinas como la pleura, el pulmón, el ángulo esplénico del colon y lesiones vasculares per se $(45,46)$. Los métodos no invasivos se prefieren a la cirugía, ya que las biopsias esplénicas por laparoscopia requieren anestesia general y están limitadas a casos que tengan lesiones visibles en la superficie del órgano. En cuanto al rendimiento diagnóstico, en un estudio publicado por Werner y colaboradores se logró el diagnóstico en casi el $70 \%$ de los pacientes con biopsias tomadas por laparoscopia (47). Respecto a las biopsias por aspiración percutáneas, el Dr. Soderstrom publicó un trabajo con más de 1000 biopsias por aspiración a ciegas, en las que no hubo complicaciones mayores (48). Posteriormente, aparecieron varios manuscritos que describen las biopsias percutáneas con aspiración por aguja fina, guiada por ecografía y TAC en enfermedades benignas y malignas esplénicas, y mostró buenos resultados en términos de eficacia y seguridad (49-51). En Italia, se realizó un estudio multicéntrico que incluyó 398 pacientes en quienes se realizaron BAAF percutáneas guiadas por ultrasonido (45). En dicho estudio se presentaron complicaciones del procedimiento en el 5,2 \% de los pacientes. Las biopsias percutáneas tienen limitaciones en pacientes obesos, con historia de intervenciones quirúrgicas abdominales y con ascitis; además, se requiere en todos los casos el servicio de radiología intervencionista con alta experiencia.

El USE para PAAF del bazo es posible gracias a la proximidad del bazo con la pared gástrica y la ventaja es la visualización en tiempo real de la aguja y de sus movimientos. Recientemente, se publicó la experiencia de un centro europeo, en la que se describió que se obtuvo un diagnóstico histopatológico concluyente en el $66,7 \%$, de los cuales la mitad se relacionó con linfoma con compromiso esplénico. Uno de los datos más relevantes de este estudio es que no se presentaron complicaciones y se concluye que el USE-PAAF esplénico es necesario o de primera opción en pacientes con sospecha de malignidad o de filiación etiológica incierta, en el escenario de esplenomegalia o lesiones ocupantes de espacio esplénicas sospechosas o de pequeño tamaño que tengan limitaciones a la toma de biopsias percutáneas (52).

\section{CONCLUSIONES}

La endosonografía tiene múltiples aplicaciones como método diagnóstico en la estadificación de tumores gastrointestinales. Es un método complementario a otras modalidades de imagen como la TAC, la RMN, PET scan en la estadificación de las neoplasias de esófago, estómago y recto. Tiene mayor rendimiento en la estadificación de la $\mathrm{T}$ (tumor) y la $\mathrm{N}$ (ganglio) para estas 3 neoplasias.

Es el método de elección en la evaluación de lesiones subepiteliales, ya que puede discriminar con bastante especificidad la ecocapa afectada, así mismo caracterizar el tipo de lesión, tamaño y la ubicación de las mismas.

Es el método con mayor rendimiento diagnóstico en lesiones pancreáticas menores de $10 \mathrm{~mm}$, aumentando su especificidad gracias a la posibilidad de tomar punción con aspiración o biopsias de la lesión.

La ablación USE-guiada es un método prometedor en el tratamiento del insulinoma pancreático funcionante en pacientes no candidatos quirúrgicos o quienes rechacen la cirugía. La otra aplicación emergente es la toma de punciones y biopsias esplénicas USE-guiadas en el escenario de pacientes con fiebre de origen desconocido, pérdida anormal de peso que tengan lesiones ocupantes de espacio o esplenomegalia no filiada. 


\section{REFERENCIAS}

1. Yasuda K. The Handbook of endoscopic ultrasonography in digestive tract. $1^{\text {st }}$ edition. New York: Blackwell; 2000.

2. Polkowski M, Larghi A, Weynand B, Boustière C, Giovannini M, Pujol B, Dumonceau JM; European Society of Gastrointestinal Endoscopy (ESGE). Learning, techniques, and complications of endoscopic ultrasound (EUS)guided sampling in gastroenterology: European Society of Gastrointestinal Endoscopy (ESGE) Technical Guideline. Endoscopy. 2012;44(2):190-205. https://doi.org/10.1055/s-0031-1291543

3. Wani S, Keswani RN, Petersen B, Edmundowicz SA, Walsh CM, Huang C, Cohen J, Cote G. Training in EUS and ERCP: standardizing methods to assess competence. Gastrointest Endosc. 2018;87(6):1371-1382. https://doi.org/10.1016/j.gie.2018.02.009

4. Eloubeidi MA, Tamhane A, Lopes TL, Morgan DE, Cerfolio RJ. Cervical esophageal perforations at the time of endoscopic ultrasound: a prospective evaluation of frequency, outcomes, and patient management. Am J Gastroenterol. 2009;104(1):53-6. https://doi.org/10.1038/ajg.2008.21

5. Mosquera-Klinger G, Torres Rincón R. Perforación iatrogénica faringoesofágica tratada con prótesis esofágica totalmente cubierta: reporte de caso. Gastroenterol Hepatol. 2019;42(7):429-30. https://doi.org/10.1016/j.gastrohep.2018.08.008

6. ASGE Standards of Practice Committee, Early DS, Acosta RD, Chandrasekhara V, Chathadi KV, Decker GA, Evans JA, Fanelli RD, Fisher DA, Fonkalsrud L, Hwang JH, Jue TL, Khashab MA, Lightdale JR, Muthusamy VR, Pasha SF, Saltzman JR, Sharaf RN, Shergill AK, Cash BD. Adverse events associated with EUS and EUS with FNA. Gastrointest Endosc. 2013;77(6):839-43. https://doi.org/10.1016/j.gie.2013.02.018

7. Standards of Practice Committee, Faulx AL, Kothari S, Acosta RD, Agrawal D, Bruining DH, Chandrasekhara V, Eloubeidi MA, Fanelli RD, Gurudu SR, Khashab MA, Lightdale JR, Muthusamy VR, Shaukat A, Qumseya BJ, Wang A, Wani SB, Yang J, DeWitt JM. The role of endoscopy in subepithelial lesions of the GI tract. Gastrointest Endosc. 2017;85(6):1117-1132. https://doi.org/10.1016/j.gie.2017.02.022

8. Polkowski M. Endoscopic ultrasound and endoscopic ultrasound- guided fine-needle biopsy for the diagnosis of malignant submucosal tumors. Endoscopy 2005;37(7):635-45. https://doi.org/10.1055/s-2005-861422

9. Nishida T, Blay JY, Hirota S, Kitagawa Y, Kang YK. The standard diagnosis, treatment, and follow-up of gastrointestinal stromal tumors based on guidelines. Gastric Cancer. 2016;19(1):3-14.

https://doi.org/10.1007/s10120-015-0526-8

10. Trindade AJ, Benias PC, Alshelleh M, Bazarbashi AN, Tharian B, Inamdar S, Sharma N, Zelt C, Korrapati P,
Barakat M, Sejpal DV, Ryou M. Fine-needle biopsy is superior to fine-needle aspiration of suspected gastrointestinal stromal tumors: a large multicenter study. Endosc Int Open. 2019;7(7):E931-E936.

https://doi.org/10.1055/a-0953-1640

11. Kim SY, Shim KN, Lee JH, Lim JY, Kim TO, Choe AR, Tae $\mathrm{CH}$, Jung HK, Moon CM, Kim SE, Jung SA. Comparison of the Diagnostic Ability of Endoscopic Ultrasonography and Abdominopelvic Computed Tomography in the Diagnosis of Gastric Subepithelial Tumors. Clin Endosc. 2019;52(6):565-573. https://doi.org/10.5946/ce.2019.019

12. SabikJF, Rice TW, Goldblum JR, Koka A, Kirby TJ, Medendorp SV, Adelstein DJ. Superficial esophageal carcinoma. Ann Thorac Surg. 1995;60(4):896-901; discussion 902. https://doi.org/10.1016/0003-4975(95)00542-s

13. Hulshoff JB, Mul VEM, de Boer HEM, Noordzij W, Korteweg T, van Dullemen HM, Nagengast WB, Oppedijk V, Pierie JPEN, Plukker JTM. Impact of Endoscopic Ultrasonography on ${ }^{18}$ F-FDG-PET/CT Upfront Towards Patient Specific Esophageal Cancer Treatment. Ann Surg Oncol. 2017;24(7):1828-1834. https://doi.org/10.1245/s10434-017-5835-1

14. Foley KG, Morgan C, Roberts SA, Crosby T. Impact of Positron Emission Tomography and Endoscopic Ultrasound Length of Disease Difference on Treatment Planning in Patients with Oesophageal Cancer. Clin Oncol (R Coll Radiol). 2017;29(11):760-766. https://doi.org/10.1016/j.clon.2017.07.014

15. Foley K, Findlay J, Goh V. Novel imaging techniques in staging oesophageal cancer. Best Pract Res Clin Gastroenterol. 2018;36-37:17-25. https://doi.org/10.1016/j.bpg.2018.11.009

16. Morgan MA, Twine CP, Lewis WG, Lambe R, Oliphant HE, Robinson M, Crosby TD, Roberts SA. Prognostic significance of failure to cross esophageal tumors by endoluminal ultrasound. Dis Esophagus. 2008;21(6):508-13. https://doi.org/10.1111/j.1442-2050.2008.00809.x

17. Sitarz R, Skierucha M, Mielko J, Offerhaus GJA, Maciejewski R, Polkowski WP. Gastric cancer: epidemiology, prevention, classification, and treatment. Cancer Manag Res. 2018;10:239-248. https://doi.org/10.2147/CMAR.S149619

18. Mocellin S, Pasquali S. Diagnostic accuracy of endoscopic ultrasonography (EUS) for the preoperative locoregional staging of primary gastric cancer. Cochrane Database Syst Rev. 2015;2015(2):CD009944. https://doi.org/10.1002/14651858.CD009944.pub2

19. Cimavilla Román M, de la Serna Higuera C, Loza Vargas LA, Benito Fernández C, Barrio Andrés J, Madrigal Rubiales B, Fernández Pérez G, Pérez-Miranda M. Endoscopic ultrasound versus multidetector computed tomography in preoperative gastric cancer staging. Rev Esp 
Enferm Dig. 2017;109(11):761-767.

https://doi.org/10.17235/reed.2017.4638/2016

20. Banerjee AK, Longcroft-Wheaton G, Beable R, Conti J, Khan J, Bhandari P. The role of imaging and biopsy in the management and staging of large non-pedunculated rectal polyps. Expert Rev Gastroenterol Hepatol. 2018; $12(8): 749-755$. https://doi.org/10.1080/17474124.2018.1492377

21. Fuchsjäger MH, Maier AG, Schima W, Zebedin E, Herbst F, Mittlböck M, Wrba F, Lechner GL. Comparison of transrectal sonography and double-contrast MR imaging when staging rectal cancer. AJR Am J Roentgenol. 2003; $181(2): 421-7$. https://doi.org/10.2214/ajr.181.2.1810421

22. Puli SR, Bechtold ML, Reddy JB, Choudhary A, Antillon MR, Brugge WR. How good is endoscopic ultrasound in differentiating various $\mathrm{T}$ stages of rectal cancer? Meta-analysis and systematic review. Ann Surg Oncol. 2009; 16(2):254-65. https://doi.org/10.1245/s10434-008-0231-5

23. Beets-Tan RGH, Lambregts DMJ, Maas M, Bipat $S$, Barbaro B, Curvo-Semedo L, Fenlon HM, Gollub MJ, Gourtsoyianni S, Halligan S, Hoeffel C, Kim SH, Laghi A, Maier A, Rafaelsen SR, Stoker J, Taylor SA, Torkzad MR, Blomqvist L. Magnetic resonance imaging for clinical management of rectal cancer: Updated recommendations from the 2016 European Society of Gastrointestinal and Abdominal Radiology (ESGAR) consensus meeting. Eur Radiol. 2018;28(4):1465-1475. https://doi.org/10.1007/s00330-017-5026-2

24. Worrell S, Horvath K, Blakemore T, Flum D. Endorectal ultrasound detection of focal carcinoma within rectal adenomas. Am J Surg. 2004;187(5):625-9; discussion 629. https://doi.org/10.1016/j.amjsurg.2004.01.005

25. Waage JE, Leh S, Røsler C, Pfeffer F, Bach SP, Havre RF, Haldorsen IS, Ødegaard S, Baatrup G. Endorectal ultrasonography, strain elastography and MRI differentiation of rectal adenomas and adenocarcinomas. Colorectal Dis. 2015;17(2):124-31. https://doi.org/10.1111/codi.12845

26. Marone P, de Bellis M, D’Angelo V, Delrio P, Passananti V, Di Girolamo E, Rossi GB, Rega D, Tracey MC, Tempesta AM. Role of endoscopic ultrasonography in the locoregional staging of patients with rectal cancer. World J Gastrointest Endosc. 2015;7(7):688-701. https://doi.org/10.4253/wjge.v7.i7.688

27. Bipat S, Glas AS, Slors FJ, Zwinderman AH, Bossuyt PM, Stoker J. Rectal cancer: local staging and assessment of lymph node involvement with endoluminal US, $\mathrm{CT}$, and MR imaging--a meta-analysis. Radiology. 2004;232(3):773-83. https://doi.org/10.1148/radiol.2323031368

28. Benson AB, Venook AP, Al-Hawary MM, Arain MA, Chen YJ, Ciombor KK, Cohen S, Cooper HS, Deming D, Garrido-Laguna I, Grem JL, Gunn A, Hoffe S, Hubbard J, Hunt S, Kirilcuk N, Krishnamurthi S, Messersmith WA,
Meyerhardt J, Miller ED, Mulcahy MF, Nurkin S, Overman MJ, Parikh A, Patel H, Pedersen K, Saltz L, Schneider C, Shibata D, Skibber JM, Sofocleous CT, Stoffel EM, StotskyHimelfarb E, Willett CG, Johnson-Chilla A, Gurski LA. NCCN Guidelines Insights: Rectal Cancer, Version 6.2020. J Natl Compr Canc Netw. 2020 Jul;18(7):806-815.

29. Benson AB, Venook AP, Al-Hawary MM, Cederquist L, Chen YJ, Ciombor KK, Cohen S, Cooper HS, Deming D, Engstrom PF, Grem JL, Grothey A, Hochster HS, Hoffe S, Hunt S, Kamel A, Kirilcuk N, Krishnamurthi S, Messersmith WA, Meyerhardt J, Mulcahy MF, Murphy JD, Nurkin S, Saltz L, Sharma S, Shibata D, Skibber JM, Sofocleous CT, Stoffel EM, Stotsky-Himelfarb E, Willett CG, Wuthrick E, Gregory KM, Gurski L, Freedman-Cass DA. Rectal Cancer, Version 2.2018, NCCN Clinical Practice Guidelines in Oncology. J Natl Compr Canc Netw. 2018;16(7):874-901. https://doi.org/10.6004/jnccn.2018.0061

30. Bhutani MS, Koduru P, Joshi V, Saxena P, Suzuki R, Irisawa A, Yamao K. The role of endoscopic ultrasound in pancreatic cancer screening. Endosc Ultrasound. 2016;5(1):8-16. https://doi.org/10.4103/2303-9027.175876

31. Uehara H, Ikezawa K, Kawada N, Fukutake N, Katayama K, Takakura R, Takano Y, Ishikawa O, Takenaka A. Diagnostic accuracy of endoscopic ultrasound-guided fine needle aspiration for suspected pancreatic malignancy in relation to the size of lesions. J Gastroenterol Hepatol. 2011;26(8):1256-61. https://doi.org/10.1111/j.1440-1746.2011.06747.x

32. Hewitt MJ, McPhail MJ, Possamai L, Dhar A, Vlavianos P, Monahan KJ. EUS-guided FNA for diagnosis of solid pancreatic neoplasms: a meta-analysis. Gastrointest Endosc. 2012;75(2):319-31. https://doi.org/10.1016/j.gie.2011.08.049

33. Ayres LR, Kmiotek EK, Lam E, Telford JJ. A Comparison of Endoscopic Ultrasound-Guided Fine-Needle Aspiration and Fine-Needle Biopsy in the Diagnosis of Solid Pancreatic Lesions. Can J Gastroenterol Hepatol. 2018;2018:1415062. https://doi.org/10.1155/2018/1415062

34. Mosquera-Klinger G, Carvajal JJ, Echeverri C, Pérez JC, Cardona R, Valencia-Maturana J, Sánchez-Garrido H. Rendimiento diagnóstico de las punciones de lesiones biliopancreáticas guiadas por ultrasonido endoscopio con patólogo en sala. En prensa.

35. Goldberg SN, Mallery S, Gazelle GS, Brugge WR. EUSguided radiofrequency ablation in the pancreas: results in a porcine model. Gastrointest Endosc. 1999;50(3):392-401. https://doi.org/10.1053/ge.1999.v50.98847

36. Wu Y, Tang Z, Fang H, Gao S, Chen J, Wang Y, Yan H. High operative risk of cool-tip radiofrequency ablation for unresectable pancreatic head cancer. J Surg Oncol. 2006;94(5):392-5. https://doi.org/10.1002/jso.20580

37. Spiliotis JD, Datsis AC, Michalopoulos NV, Kekelos SP, Vaxevanidou A, Rogdakis AG, Christopoulou AN. 
Radiofrequency ablation combined with palliative surgery may prolong survival of patients with advanced cancer of the pancreas. Langenbecks Arch Surg. 2007;392(1):55-60. https://doi.org/10.1007/s00423-006-0098-5

38. Elias D, Baton O, Sideris L, Lasser P, Pocard M. Necrotizing pancreatitis after radiofrequency destruction of pancreatic tumours. Eur J Surg Oncol. 2004;30(1):85-7. https://doi.org/10.1016/j.ejso.2003.10.013

39. Lakhtakia S, Ramchandani M, Galasso D, Gupta R, Venugopal S, Kalpala R, Reddy DN. EUS-guided radiofrequency ablation for management of pancreatic insulinoma by using a novel needle electrode (with videos). Gastrointest Endosc. 2016;83(1):234-9. https://doi.org/10.1016/j.gie.2015.08.085

40. Waung JA, Todd JF, Keane MG, Pereira SP. Successful management of a sporadic pancreatic insulinoma by endoscopic ultrasound-guided radiofrequency ablation. Endoscopy. 2016;48 Suppl 1:E144-5. https://doi.org/10.1055/s-0042-104650

41. Limmer S, Huppert PE, Juette V, Lenhart A, Welte M, Wietholtz H. Radiofrequency ablation of solitary pancreatic insulinoma in a patient with episodes of severe hypoglycemia. Eur J Gastroenterol Hepatol. 2009;21(9):1097-101. https://doi.org/10.1097/MEG.0b013e328323d70e

42. Pai M, Habib N, Senturk H, Lakhtakia S, Reddy N, Cicinnati VR, Kaba I, Beckebaum S, Drymousis P, Kahaleh M, Brugge W. Endoscopic ultrasound guided radiofrequency ablation, for pancreatic cystic neoplasms and neuroendocrine tumors. World J Gastrointest Surg. 2015;7(4):52-9. https://doi.org/10.4240/wjgs.v7.i4.52

43. Rossi S, Viera FT, Ghittoni G, Cobianchi L, Rosa LL, Siciliani L, Bortolotto C, Veronese L, Vercelli A, Gallotti A, Ravetta V. Radiofrequency ablation of pancreatic neuroendocrine tumors: a pilot study of feasibility, efficacy, and safety. Pancreas. 2014;43(6):938-45. https://doi.org/10.1097/MPA.0000000000000133

44. Mosquera-Klinger G, Carvajal JJ. Insulinoma pancreático sintomático: Tratamiento ablativo mediante etanolización guiado por ultrasonido endoscópico. Rev Esp Enf Dig. En prensa, 2020.

https://doi.org/10.17235/reed.2020.7109/2020

45. Civardi G, Vallisa D, Bertè R, Giorgio A, Filice C, Caremani M, Caturelli E, Pompili M, De Sio I, Buscarini E, Cavanna L. Ultrasound-guided fine needle biopsy of the spleen: high clinical efficacy and low risk in a multicenter Italian study. Am J Hematol. 2001;67(2):93-9. https://doi.org/10.1002/ajh.1085

46. Quinn SF, vanSonnenberg E, Casola G, Wittich GR, Neff CC. Interventional radiology in the spleen. Radiology. 1986;161(2):289-91. https://doi.org/10.1148/radiology.161.2.3763890

47. Werner T, Koch J, Frenzel C, Lohse AW, Denzer UW. Effectiveness and safety of minilaparoscopy-guided spleen biopsy: a retrospective series of 57 cases. Surg Endosc. 2012;26(9):2416-22. https://doi.org/10.1007/s00464-012-2190-y

48. Söderström N. How to use cytodiagnostic spleen puncture. Acta Med Scand. 1976;199(1-2):1-5. https://doi.org/10.1111/j.0954-6820.1976.tb06683.x

49. Solbiati L, Bossi MC, Bellotti E, Ravetto C, Montali G. Focal lesions in the spleen: sonographic patterns and guided biopsy. AJR Am J Roentgenol. 1983;140(1):59-65. https://doi.org/10.2214/ajr.140.1.59

50. Silverman JF, Geisinger KR, Raab SS, Stanley MW. Fine needle aspiration biopsy of the spleen in the evaluation of neoplastic disorders. Acta Cytol. 1993;37(2):158-62.

51. Zeppa P, Vetrani A, Luciano L, Fulciniti F, Troncone G, Rotoli B, Palombini L. Fine needle aspiration biopsy of the spleen. A useful procedure in the diagnosis of splenomegaly. Acta Cytol. 1994;38(3):299-309.

52. Mosquera-Klinger G, de la Serna Higuera C, Bazaga S, García-Alonso FJ, Sánchez Ocaña R, Antolín Melero B, de Benito Sanz M, Madrigal B, Torres Á, Pérez-Miranda M. Endoscopic ultrasound-guided fine-needle aspiration for splenomegaly and focal splenic lesion: is it safe, effective and necessary? Rev Esp Enferm Dig. 2020;112(5):355-359. https://doi.org/10.17235/reed.2020.6667/2019 